\section{CONDIÇÃO NUTRICIONAL DE CRIANÇAS ADMITIDAS EM UMA INSTITUIÇÃO DE ACOLHIMENTO}

\author{
Nutritional status of children admitted to a shelter \\ Condición nutricional de niños admitidos en una institución \\ de acogida
}

\section{RESUMO}

Objetivo: Avaliar o estado nutricional de crianças de uma instituição de acolhimento na cidade de Recife, no momento da admissão. Métodos: Estudo transversal, retrospectivo, que estudou uma amostra de 166 crianças menores de cinco anos de idade, acolhidas entre setembro de 2007 e setembro de 2012, em uma instituição de Recife, Pernambuco. As variáveis estudadas foram sexo, peso, estatura, idade e motivo do acolhimento. O estado nutricional foi analisado mediante os índices antropométricos: peso por idade, estatura por idade, peso por estatura e índice de massa corporal para idade pelo Escore Z, utilizando como padrão de referência as curvas de crescimento da Organização Mundial da Saúde. Para as análises estatísticas, utilizou-se o software SPSS, versão 13.0. Para todas as análises, considerou-se significância estatística quando $\mathrm{p}<0,05$. Resultados: A maioria das crianças (78,3\%) chegou eutrófica à instituição, entretanto, os níveis de desnutrição $(9,6 \%)$ foram superiores aos da população de mesma faixa etária no estado de Pernambuco $(3,2 \%)$. A desnutrição segundo o índice peso por estatura foi maior na faixa etária dos 6 aos 11 meses ( $23 \%)$, mas somente com esse índice a associação com a faixa etária foi positiva $(\mathrm{p}=0,025)$. A prevalência de baixa estatura foi de $12 \%$. Conclusão: A desnutrição e a baixa estatura na amostra estudada apresentaram índices maiores que o encontrado em crianças de mesma faixa etária do Estado de Pernambuco, e estão aquém dos valores aceitáveis por organizações internacionais.

Descritores: Criança; Abrigo; Avaliação Nutricional.

\section{ABSTRACT}

Objective: To assess the nutritional status of children in a shelter located in the city of Recife, Northeastern Brazil. Methods: Retrospective cross-sectional study carried out with a sample of 166 children under five years of age admitted to a shelter in Recife, Pernambuco, between September 2007 and September 2012. The study variables were: gender, weight, height, age and reason for institutionalization. Nutritional status was determined based on the following anthropometric indicators: weight-for-age, height-for-age, weight-for-height and body mass index-for-age using the $Z$ score and the World Health Organization growth curves standards. SPSS 13.0 was used for the statistical analysis. For all analyses, statistical significance was set at $p<0.05$. Results: Most children (78.3\%) were at normal weight at admission; however, the underweight rate (9.6\%) was higher than that of the population of the same age group in the state of Pernambuco (3.2\%). Based on the weight-for-height index, underweight was higher in the age group 6-to-11 months (23\%), and it was positively associated with age $(p=0.025)$. The prevalence of stunting was $12 \%$. Conclusion: Underweight and stunting rates found in the study sample were higher than those reported for children in the same age group in the state of Pernambuco and are much higher than the recommendations of international organizations.

Descriptors: Child; Shelter; Nutrition Assessment.
Artigo Original
1) Maternidade Escola Assis Chateaubriand da Universidade Federal do Ceará - MEAC/ UFC - Fortaleza (CE) - Brasil

2) Universidade Federal de Pernambuco UFPE - Recife (PE) - Brasil

3) Faculdade Maurício de Nassau UNINASSAU - Recife (PE) - Brasil
Recebido em: 17/11/2015 Revisado em: 20/12/2015 Aceito em: 17/02/2016 


\section{RESUMEN}

Objetivo: Evaluar el estado nutricional de niños de una institución de acogida localizada en la ciudad de Recife, noreste brasileño. Métodos: Estudio transversal y retrospectivo que estudió una muestra de 166 niños abajo de cinco años de edad acogidos entre septiembre de 2007 y septiembre de 2012 en una institución de Recife, Pernambuco. Las variables estudiadas fueron el sexo, el peso, la estatura, la edad y el motivo de la acogida. El estado nutricional fue analizado a través de los índices antropométricos: el peso por la edad, la estatura por la edad, el peso por la estatura y el índice de masa corporal para la edad por la puntuación $Z$ utilizando como patrón de referencia las curvas de crecimiento de la Organización Mundial de la Salud. Para los análisis estadisticos se utilizó el software SPSS, versión 13.0. Para todos los análisis se consideró la significancia estadística para $p<0,05$. Resultados: La mayoría de los niños (78,3\%) llegó eutrófica a la institución, sin embargo, los niveles de desnutrición (9,6\%) fueron superiores a los de la población para la misma franja de edad en el estado de Pernambuco (3,2\%). La desnutrición según el índice del peso por la estatura fue mayor para la franja de edad entre los 6 y 11 meses (23\%) pero solamente con ese índice la asociación con la franja de edad fue positiva $(p=0,025)$. La prevalencia de baja estatura fue del 12\%. Conclusión: La desnutrición y la baja estatura de la muestra estudiada presentaron mayores índices que aquellos encontrados en los niños de la misma franja de edad del estado de Pernambuco y están inferiores a los valores aceptables por las organizaciones internacionales.

Descriptores: Niño; Refugio; Evaluación Nutricional.

\section{INTRODUÇÃO}

No Brasil, cerca de 30 mil crianças e adolescentes vivem em instituições de acolhimento, principalmente devido a condições socioeconômicas desfavoráveis ${ }^{(1)}$, e isso muitas vezes se reflete em um déficit nutricional, principalmente nas crianças menores de cinco anos ${ }^{(2)}$. O estado nutricional da criança reflete suas condições de vida, pois o crescimento é sensível a diversos fatores, como condições ambientais, situação econômica e de habitação precárias, baixa escolaridade materna, ausência da assistência pré-natal e ocorrência de baixo peso ao nascer ${ }^{(3)}$.

A desnutrição ainda é considerada um problema de saúde pública no Brasil, entretanto, vem apresentando significativa diminuição de sua prevalência nas últimas décadas, embora haja diferença em sua magnitude entre as regiões do país, sendo a prevalência da região Nordeste $(17,9 \%)$ muito superior à da região do Centro-Sul $(5,6 \%)^{(4)}$. O processo de transição nutricional no Brasil revela como consequência as transformações sociais que resultaram na diminuição da pobreza no país, gerando impacto positivo na redução da desnutrição, assim como da baixa estatura $^{(2,5)}$. Em Pernambuco, particularmente, observa-se uma queda nos déficits nutricionais ao longo do tempo ${ }^{(6,7)}$. O déficit nutricional na infância pode levar a consequências irreversíveis para o indivíduo, sobretudo para o seu desenvolvimento intelectual ${ }^{(8)}$.

De acordo com o Estatuto da Criança e do Adolescente, o direito à vida, à saúde, ao respeito e à dignidade é considerado fundamental, portanto, quando esses princípios básicos não são assegurados, as instituições de acolhimento devem assumir o papel da família para a garantia desses direitos, oferecendo condições adequadas para o crescimento e o desenvolvimento normais. $\mathrm{O}$ acolhimento, considerado medida de proteção, deve ser temporário ${ }^{(9)}$, entretanto, muitas crianças podem permanecer nesta condição por vários $\operatorname{anos}^{(10)}$.

As instituições de acolhimento devem se responsabilizar integralmente pelo crescimento e desenvolvimento neuropsicomotor das crianças sob sua proteção. A capacidade cognitiva da criança depende da qualidade da estimulação do ambiente ${ }^{(11,12)}$. Estudos revelaram os efeitos negativos da institucionalização no desenvolvimento infantil ${ }^{(13,14)}$. $\mathrm{O}$ estado nutricional de crianças que se encontram institucionalizadas pode e deve ser monitorado por meio da avaliação antropométrica, método simples, de baixo custo e sensível para detectar os distúrbios nutricionais. O monitoramento poderá ser feito utilizando-se a própria Caderneta de Saúde da Criança, pois é um instrumento destinado para essa finalidade ${ }^{(15)}$.

Poucos estudos documentam a condição nutricional da criança na admissão nessas instituições, bem como os efeitos da institucionalização no seu desenvolvimento físico $^{(16,17)}$. Nesse contexto, o objetivo deste trabalho foi avaliar o estado nutricional de crianças de uma instituição de acolhimento na cidade de Recife, no momento da admissão.

\section{MÉTODOS}

Trata-se de um estudo transversal, retrospectivo, realizado em uma instituição de acolhimento para crianças em risco social, localizada na cidade de Recife, PE/Brasil. Constituíram a amostra 166 crianças menores de 60 meses, de ambos os sexos, admitidas no período de setembro de 2007 a setembro de 2012. Este estudo é resultado de um levantamento de cinco anos sobre o estado nutricional no momento da admissão das crianças.

A instituição à qual a amostra estudada pertence é uma Organização Não Governamental (ONG), reconhecida como entidade pública que acolhe temporariamente crianças em situação de risco social ou abandono, promovendo sua proteção integral até a decisão judicial sobre seu destino - reintegração familiar ou sua colocação, por adoção, em família substituta. Ela possui uma equipe 
multidisciplinar, com pediatra, nutricionista, técnicos de enfermagem, pedagogos, psicólogos e assistentes sociais. Acolhe predominantemente crianças da capital, sendo rara a inclusão de crianças de outros municípios do estado.

Adotaram-se como critérios de exclusão: prematuridade, baixo peso ao nascer, síndrome genética ou doença crônica grave que pudessem levar ao déficit nutricional, paralisia cerebral, desconhecimento da data de nascimento da criança, impossibilidade de aferição das medidas antropométricas nas primeiras $48 \mathrm{~h}$ de admissão e readmissões por devolução de guarda.

Das 206 crianças menores de cinco anos admitidas na instituição, entre setembro de 2007 e setembro de 2012, $40(19,4 \%)$ tiveram que ser excluídas. Destas, 15 (37,5\%) por prematuridade, $12(30,0 \%)$ pela impossibilidade de aferição das medidas antropométricas nas primeiras $48 \mathrm{~h}$ de admissão, 8 (20,0\%) por serem readmitidas por devolução de guarda, $4(10,0 \%)$ por serem maiores de 5 anos de idade, e $1(2,5 \%)$ por desconhecimento da data de seu nascimento.

As variáveis estudadas foram: sexo, idade, peso, estatura e motivo do abrigamento da criança na instituição; e os dados foram obtidos através dos formulários individuais das crianças. Considerou-se a termo a criança nascida a partir de $37^{\mathrm{a}}$ semana de gestação; e de baixo peso ao nascer aquelas nascidas com peso $<2500 \mathrm{~g}^{(18)}$. Para o diagnóstico nutricional, utilizou-se o método antropométrico, adotandose os seguintes índices: peso por idade $(\mathrm{P} / \mathrm{I})$, peso por estatura $(\mathrm{P} / \mathrm{E})$, estatura por idade $(\mathrm{E} / \mathrm{I})$ e índice de massa corporal $\left(\mathrm{kg} / \mathrm{m}^{2}\right)$ por idade (IMC/I), expressos em Escore $Z$, considerando-se os pontos de corte da Organização Mundial da Saúde ${ }^{(19)}$ estabelecidos para crianças menores de 60 meses.

O índice estatura por idade (E/I) foi empregado como indicador de baixa estatura e o índice de massa corporal $\left(\mathrm{kg} / \mathrm{m}^{2}\right)$ por idade (IMC/I), como indicador do estado nutricional atual. Para a classificação do estado nutricional segundo os índices antropométricos escolhidos, adotouse a recomendação do Sistema de Vigilância Alimentar e Nutricional (SISVAN) ${ }^{(15)}$, que define para os índices P/E e IMC/I: desnutrição (magreza e magreza acentuada), quando escore-Z $<-2$; eutrofia, quando Escore $Z$ for $\geq-2$ e $\leq+2$ e excesso de peso (sobrepeso e obesidade), quando Escore $Z$ $>+2$. Para o índice E/I: baixa estatura, quando $<$ Escore $Z$ -2; estatura adequada, quando Escore $Z \geq-2$. E para o índice P/I: baixo peso, quando Escore $Z<-2$; peso adequado, quando Escore $Z$ for $\geq-2$ e $\leq+2$; e peso elevado, quando Escore $\mathrm{Z}$ for $\geq+2$.

A estatura e o peso das crianças são rotineiramente aferidos por uma nutricionista no momento da admissão na instituição, seguindo técnicas preconizadas pelo Ministério da Saúde ${ }^{(15)}$. A instituição dispõe de equipamentos adequados para a verificação das medidas antropométricas, como balança digital pediátrica com capacidade máxima de $15 \mathrm{~kg}$ e graduação de $5 \mathrm{~g}$ para pesar crianças até 24 meses e balança digital plataforma com capacidade máxima de 180 $\mathrm{kg}$ e graduação de $100 \mathrm{~g}$ para os maiores de 24 meses, ambas da marca Filizola, além de régua antropométrica pediátrica de madeira com marcador removível, escala de $100 \mathrm{~cm}$ e graduação em milímetros, numeradas a cada centímetro.

Os motivos de acolhimento da criança na instituição foram classificados de acordo com a categorização do Núcleo de Orientação e Fiscalização de Entidades (NOFE), do Centro Integrado da Criança e do Adolescente da $1^{\mathrm{a}}$ Vara da Infância e da Juventude de Pernambuco: abandono, violência doméstica, violência social (mendicância e miserabilidade) e situação de rua. Além deles, também foram incluídas crianças admitidas através do Programa Mãe Legal, desenvolvido pelo Núcleo de Curadoria Especial e Proteção à Família (NUCE) da $2^{\mathrm{a}}$ Vara da Infância e Juventude do Recife. Para efeito de análise, as crianças abandonadas e provenientes do Programa Mãe Legal foram agrupadas.

As análises estatísticas foram realizadas no software SPSS, versão 13.0 (SPSS Inc., Chicago, IL, USA), e as variáveis contínuas foram testadas quanto à normalidade de distribuição pelo teste de Kolmogorov-Smirnov - como apresentaram distribuição normal, foram descritas na forma de média e desvio padrão. Utilizou-se o teste do Quiquadrado para comparação das variáveis categóricas, e o teste Kappa foi empregado para avaliar a concordância do diagnóstico de desnutrição estabelecido pelos índices IMC/I e o P/E. Para todas as análises, considerou-se significância estatística quando $\mathrm{p}<0,05$.

Pesquisa realizada após aprovação do Comitê de Ética do Centro Universitário Maurício de Nassau, sob protocolo $\mathrm{n}^{\circ}$ 91.170/12.

\section{RESULTADOS}

A amostra efetivamente estudada foi constituída de 166 crianças, com média de idade de $13( \pm 13,97)$ meses, variando de 0 a 53 meses. Observou-se distribuição homogênea entre os sexos, sendo 88 (53\%) meninos, e maior proporção de crianças menores de um ano de idade $(57,2 \%, \mathrm{n}=95)$, sendo $24,1 \%(\mathrm{n}=40)$ admitidas recém-nascidas.

A distribuição das crianças segundo a faixa etária revelou que $41,6 \%(n=69)$ tinham entre 0 e 5 meses de idade, e 77,1\% (n=128), até 24 meses. Com relação aos motivos de abrigamento, 98 (59\%) precisaram ser institucionalizadas devido ao abandono, $54(32,5 \%)$ por violência doméstica, $10(6 \%)$ por situação de rua e $4(2,5 \%)$ através do Programa Mãe Legal. 
Ao analisar os indicadores antropométricos, verificouse que a maioria $(78,3 \%, n=130)$ das crianças apresentavase eutrófica em todos os índices analisados. A baixa estatura foi o distúrbio nutricional mais prevalente $(12 \%, n=20)$. O IMC/I se mostrou mais sensível na detecção dos extremos da normalidade, comparando-se com o índice P/E (Figura 1). A concordância do diagnóstico de desnutrição pelos índices $\mathrm{P} / \mathrm{E}(7,2 \%, \mathrm{n}=12)$ e $\mathrm{IMC} / \mathrm{I}(9,0 \%, \mathrm{n}=15)$ foi 0,557 $(\mathrm{p}<0,001)$, considerada regular.

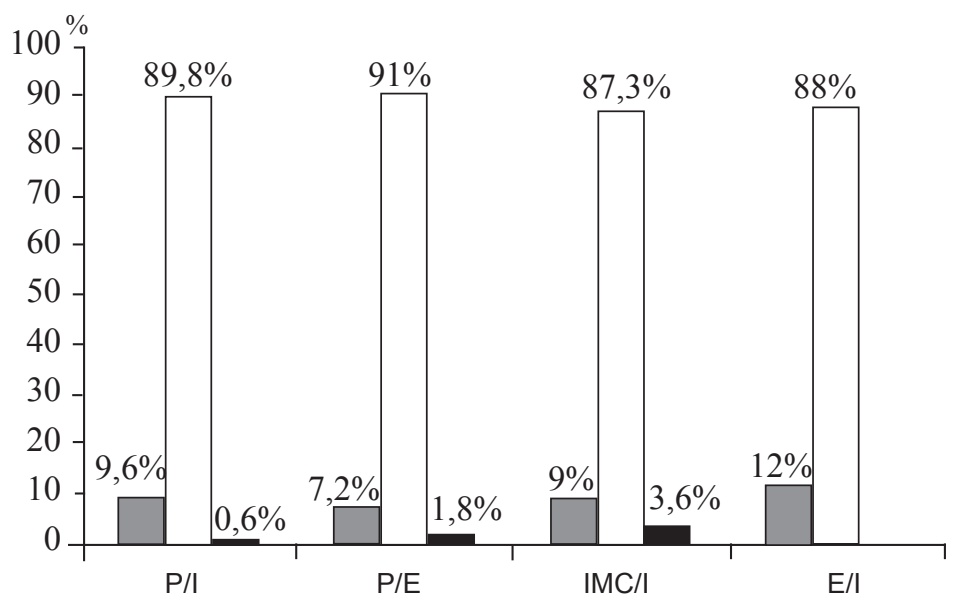

Figura 1 - Estado nutricional, segundo Escore Z, de crianças menores de cinco anos admitidas em uma instituição de acolhimento no período de 2007 a 2012. Recife, Pernambuco.

Tabela I - Estado nutricional pelo índice peso por estatura (P/E) segundo sexo, faixa etária e motivo de abrigamento de crianças menores de cinco anos admitidas em uma instituição de acolhimento no período de 2007 a 2012 . Recife, Pernambuco.

\begin{tabular}{|c|c|c|c|c|c|}
\hline \multirow{4}{*}{ Características } & \multirow{4}{*}{ Total } & \multicolumn{3}{|c|}{$\mathbf{P} / \mathbf{E}($ Escore $\mathbf{Z}) \#$} & \multirow{4}{*}{ p-valor } \\
\hline & & \multirow{2}{*}{$\begin{array}{c}\text { Magreza } \\
<-2\end{array}$} & \multirow{2}{*}{$\begin{array}{c}\text { Eutrofia } \\
-2 \text { e }<2\end{array}$} & \multirow{2}{*}{$\begin{array}{c}\text { Excesso } \\
\geq 2\end{array}$} & \\
\hline & & & & & \\
\hline & & n (\%) & n (\%) & n (\%) & \\
\hline \multicolumn{6}{|l|}{ Sexo } \\
\hline Masculino & 88 & $6(7,0 \%)$ & $80(91,0 \%)$ & $2(2,0 \%)$ & \multirow[b]{2}{*}{0,87} \\
\hline Feminino & 78 & $6(8,0 \%)$ & $71(91,0 \%)$ & $1(1,0 \%)$ & \\
\hline \multicolumn{6}{|l|}{ Faixa etária } \\
\hline $0-5$ meses & 69 & $3(4,0 \%)$ & $66(96,0 \%)$ & $0(0 \%)$ & \multirow{4}{*}{$0,02 *$} \\
\hline 6-11 meses & 26 & $6(23,0 \%)$ & $19(73,0 \%)$ & $1(4,0 \%)$ & \\
\hline $12-23$ meses & 32 & $2(6,0 \%)$ & $29(91,0 \%)$ & $1(3,0 \%)$ & \\
\hline$\geq 24$ meses & 39 & $1(2,6 \%)$ & $37(95,0 \%)$ & $1(2,6 \%)$ & \\
\hline \multicolumn{6}{|c|}{ Motivo de abrigamento } \\
\hline Abandono & 102 & $9(9,0 \%)$ & $91(89,0 \%)$ & $2(2,0 \%)$ & \multirow{3}{*}{0,80} \\
\hline Violência & 54 & $3(5,6 \%)$ & $50(92,6 \%)$ & $1(1,9 \%)$ & \\
\hline Situação de rua & 10 & $0(0 \%)$ & $10(100 \%)$ & $0(0 \%)$ & \\
\hline
\end{tabular}

P/E: Índice peso por estatura. \#Organização Mundial da Saúde, 2006.

${ }^{*} \mathrm{p}<0,05$; Teste do Qui-quadrado de Pearson 
Tabela II - Estado nutricional pelo índice de massa corporal por idade (IMC/I) segundo sexo, faixa etária e motivo de abrigamento de crianças menores de cinco anos admitidas em uma instituição de acolhimento no período de 2007 a 2012. Recife, Pernambuco.

\begin{tabular}{|c|c|c|c|c|c|}
\hline \multirow{4}{*}{ Características } & \multirow{4}{*}{ Total } & \multicolumn{3}{|c|}{ IMC/I (Escore Z)\# } & \multirow{4}{*}{ p-valor } \\
\hline & & Magreza & Eutrofia & Excesso & \\
\hline & & $<-2$ & $-2 \mathrm{e}<2$ & $\geq 2$ & \\
\hline & & n (\%) & n (\%) & n (\%) & \\
\hline \multicolumn{6}{|l|}{ Sexo } \\
\hline Masculino & 88 & $8(9,0 \%)$ & $75(85,0 \%)$ & $5(6,0 \%)$ & \multirow{2}{*}{0,31} \\
\hline Feminino & 78 & $7(9,0 \%)$ & $70(90,0 \%)$ & $1(1,0 \%)$ & \\
\hline \multicolumn{6}{|l|}{ Faixa etária } \\
\hline $0-5$ meses & 69 & $7(10,0 \%)$ & $62(90,0 \%)$ & $0(0,0 \%)$ & \multirow{4}{*}{0,10} \\
\hline 6-11 meses & 26 & $5(19,0 \%)$ & $19(73,0 \%)$ & $2(8,0 \%)$ & \\
\hline 12-23 meses & 32 & $2(6,3 \%)$ & $28(87,5 \%)$ & $2(6,3 \%)$ & \\
\hline$\geq 24$ meses & 39 & $1(3,0 \%)$ & $36(92,0 \%)$ & $2(5,0 \%)$ & \\
\hline \multicolumn{6}{|c|}{ Motivo de abrigamento } \\
\hline Abandono & 102 & $10(10,0 \%)$ & $88(86,0 \%)$ & $4(4,0 \%)$ & \multirow{3}{*}{0,95} \\
\hline Violência & 54 & $4(7,0 \%)$ & $48(89,0 \%)$ & $2(4,0 \%)$ & \\
\hline Situação de rua & 10 & $1(10,0 \%)$ & $9(90,0 \%)$ & $0(0 \%)$ & \\
\hline
\end{tabular}

IMC/I: Índice de massa corporal por idade. \#Organização Mundial da Saúde, 2006 p $>0,05$; Teste do Qui-quadrado de Pearson

Tabela III - Estado nutricional pelo índice peso por idade (P/I) segundo sexo, faixa etária e motivo de abrigamento de crianças menores de cinco anos admitidas em uma instituição de acolhimento no período de 2007 a 2012. Recife, Pernambuco.

\begin{tabular}{|c|c|c|c|c|c|}
\hline \multirow{4}{*}{ Características } & \multirow{4}{*}{ Total } & \multicolumn{3}{|c|}{ P/I (Escore Z)\# } & \multirow{4}{*}{ p-valor } \\
\hline & & Baixo & Adequado & Elevado & \\
\hline & & $<-2$ & $\geq-2$ e $\leq 2$ & $\geq 2$ & \\
\hline & & n (\%) & n (\%) & n (\%) & \\
\hline \multicolumn{6}{|l|}{ Sexo } \\
\hline Masculino & 88 & $7(8,0 \%)$ & $81(92 \%)$ & $0(0 \%)$ & \multirow{2}{*}{0,40} \\
\hline Feminino & 78 & $9(11,5 \%)$ & $68(87,2 \%)$ & $1(1,3 \%)$ & \\
\hline \multicolumn{6}{|l|}{ Faixa etária } \\
\hline $0-5$ meses & 69 & $7(10 \%)$ & $62(90 \%)$ & $0(0 \%)$ & \multirow{4}{*}{0,25} \\
\hline 6-11 meses & 26 & $4(15 \%)$ & $22(85,0 \%)$ & $0(0 \%)$ & \\
\hline $12-23$ meses & 32 & $4(12,5 \%)$ & $27(84,4 \%)$ & $1(3,1 \%)$ & \\
\hline$\geq 24$ meses & 39 & $1(3,0 \%)$ & $38(97,0 \%)$ & $0(0 \%)$ & \\
\hline \multicolumn{6}{|c|}{ Motivo de abrigamento } \\
\hline Abandono & 102 & $8(8,0 \%)$ & $94(92,0 \%)$ & $0(0,0 \%)$ & \multirow{3}{*}{0,42} \\
\hline Violência & 54 & $6(11,0 \%)$ & $47(87,0 \%)$ & $1(2,0 \%)$ & \\
\hline Situação de rua & 10 & $2(20,0 \%)$ & $8(80,0 \%)$ & $0(0,0 \%)$ & \\
\hline
\end{tabular}

P/I: Índice peso por idade. \#Organização Mundial da Saúde, 2006 p>0,05; Teste do Qui-quadrado de Pearson. 
Tabela IV - Estado nutricional pelo índice estatura por idade (E/I) segundo sexo, faixa etária e motivo de abrigamento de crianças menores de cinco anos admitidas em uma instituição de acolhimento no período de 2007 a 2012. Recife, Pernambuco.

\begin{tabular}{|c|c|c|c|c|}
\hline \multirow{4}{*}{ Características } & \multirow{4}{*}{ Total } & \multicolumn{2}{|c|}{ E/I (Escore Z)\# } & \multirow{4}{*}{ p-valor } \\
\hline & & Baixa & Adequada & \\
\hline & & $<-2$ & $\geq 2$ & \\
\hline & & n (\%) & n (\%) & \\
\hline \multicolumn{5}{|l|}{ Sexo } \\
\hline Masculino & 88 & $11(12,5 \%)$ & $77(87,5 \%)$ & \multirow{2}{*}{0,84} \\
\hline Feminino & 78 & $9(11,5 \%)$ & $69(88,5 \%)$ & \\
\hline \multicolumn{5}{|l|}{ Faixa etária } \\
\hline $0-5$ meses & 69 & $8(12,0 \%)$ & $61(88,0 \%)$ & \multirow{4}{*}{0,91} \\
\hline 6-11 meses & 26 & $3(11,5 \%)$ & $23(88,5 \%)$ & \\
\hline $12-23$ meses & 32 & $5(16,0 \%)$ & $27(84,0 \%)$ & \\
\hline$\geq 24$ meses & 39 & $4(10,0 \%)$ & $35(90 \%)$ & \\
\hline \multicolumn{5}{|c|}{ Motivo de abrigamento } \\
\hline Abandono & 102 & $8(8,0 \%)$ & $94(92,0 \%)$ & \multirow{3}{*}{0,10} \\
\hline Violência & 54 & $10(18,5 \%)$ & $44(81,5 \%)$ & \\
\hline Situação de rua & 10 & $2(20,0 \%)$ & $8(80,0 \%)$ & \\
\hline
\end{tabular}

E/I: Índice estatura por idade. \#Organização Mundial da Saúde, 2006

$\mathrm{p}>0,05$; Teste do Qui-quadrado de Pearson

\section{DISCUSSÃO}

Os recém-nascidos representaram um quarto da amostra, e isso implica na necessidade de a instituição estar preparada para acolher adequadamente essas crianças. Nessa faixa etária, o indivíduo se encontra em maior dependência do adulto, apresentando maior vulnerabilidade ao ambiente, o qual exerce importante influência sobre o crescimento e o desenvolvimento, demandando cuidados especiais. Vale ressaltar que a separação do binômio mãe-filho, nessa idade, pode acarretar enormes prejuízos à saúde, ao crescimento físico e ao desenvolvimento intelectual, devido ao desmame precoce $^{(20)}$. A instituição cujas crianças foram estudadas é uma das poucas no município que recebe crianças recémnascidas; talvez por essa razão a prevalência nessa faixa etária tenha sido alta.

Observou-se um percentual discretamente maior de crianças do sexo masculino (53\%), assim como observado em outros estudos sobre crianças em situação de acolhimento $^{(16,17)}$, embora com faixa etária diversa.

Diferentemente dos resultados deste estudo, em que $59,0 \%$ das crianças precisaram ser institucionalizadas devido ao abandono, outros autores verificaram que esta foi a segunda causa de institucionalização ${ }^{(17,21)}$. Essas diferenças acerca dos motivos de acolhimento de crianças e adolescentes no Brasil podem refletir a ausência de padronização na nomenclatura e na classificação desses motivos.

Assim como em outras pesquisas que estudaram crianças institucionalizadas, a maioria $(78,3 \%)$ das crianças encontrava-se eutrófica ${ }^{(16,17)}$. A prevalência de baixa estatura e a obesidade constituem os principais distúrbios nutricionais em crianças menores de cinco anos em Pernambuco, segundo a última pesquisa estadual ${ }^{(7)}$. No entanto, a obesidade foi um problema pouco prevalente na amostra estudada. Inclusive, ao se analisar a relação obesidade/baixo peso, que permite inferir sobre o avanço do predomínio da obesidade sobre a prevalência de baixo peso para idade ${ }^{(22)}$, observou-se uma relação de 0,06:1, expressivamente inferior à encontrada em outro estudo $(1,7: 1)$ que avaliou crianças em faixa etária similar ${ }^{(23)}$. Tal achado sugere que o processo de transição nutricional na amostra estudada não está acompanhando as mudanças experimentadas nas últimas décadas pela população brasileira.

O baixo peso representou um problema nutricional relevante na amostra estudada, com percentual superior $(9,6 \%)$ ao esperado para uma população que vive em boas condições ambientais $(2,3 \%)^{(24)}$. A prevalência de desnutrição pelos índices P/E e IMC/I foi de 7,2\% e 9,0\%, respectivamente - concordância considerada regular $(0,557 ; \mathrm{p}<0,001)$, o que demonstra a importância da adoção de vários índices na avaliação nutricional da criança. A desnutrição segundo o índice $\mathrm{P} / \mathrm{E}$ foi superior (23\%) na faixa etária de 6 aos 11 meses $(\mathrm{p}<0,025)$, fase que corresponde ao início da alimentação complementar, embora este dado não tenha sido coletado nesta pesquisa. Esse índice expressa o emagrecimento ou excesso de peso da criança, representando a relação entre o peso e a estatura, independentemente da idade. Nessa faixa etária, há necessidade de atenção especial para a garantia do crescimento e desenvolvimento 
adequados, uma vez que nela ocorrem vários marcos do desenvolvimento infantil, como sentar, engatinhar, além das primeiras erupções dentárias ${ }^{(20)}$. Além disso, a condição nutricional nessa faixa etária pode ser determinante para o estado nutricional futuro ${ }^{(25)}$.

O percentual de baixa estatura (12\%) foi superior ao observado em crianças de mesma faixa etária na população brasileira, segundo dados da última pesquisa nacional $(7 \%)^{(4)}$, e menor que a encontrada em outro estudo $(27,3 \%)^{(17)}$, que também avaliou crianças institucionalizadas, embora tenha sido utilizado o percentil para avaliação nutricional ao invés do Escore $\mathrm{Z}$ adotado neste estudo. Entretanto, foi semelhante à encontrada em outra pesquisa, também com crianças menores de cinco anos, no município de São João do Tigre, na Paraíba (16,8\% na zona rural e $12,9 \%$ na urbana), onde esse índice esteve associado significativamente com a renda familiar per capita e a escolaridade materna ${ }^{(26)}$. Vale ressaltar que o Îndice de Desenvolvimento Humano (IDH) desse município é baixo $(0,552)$, diferentemente do Recife, que possui IDH alto $(0,7772)$. Esse índice mede o progresso de uma localidade a partir de renda, saúde e educação, e seus valores variam de 0 a $1^{(2)}$. Esse achado sugere má distribuição de renda no Município do Recife, reforçando a provável vulnerabilidade social à qual as crianças estudadas podem estar expostas, uma vez que apresentam percentuais de baixa estatura similares a crianças imersas em piores condições de desenvolvimento.

Vários inquéritos nacionais demonstram diminuição expressiva da prevalência da baixa estatura em crianças menores de cinco anos, entre $1974-1975$ e $2006-2007^{(2)}$. O comprometimento do crescimento linear ocorre após longos períodos de ingestão nutricional inadequada ${ }^{(27)}$, e sua prevalência é um indicador útil das condições de vida de uma população ${ }^{(2)}$, o que pode sugerir que a amostra estudada estivesse vivendo em precárias condições anteriormente à admissão na instituição. A baixa estatura na infância está associada ao comprometimento do desenvolvimento intelectual, repercutindo em menor grau de escolaridade e, consequentemente, gerando dificuldades para o ingresso no mercado de trabalho formal na idade $\operatorname{adulta}^{(2)}$, o que, para populações como a estudada, se configura como risco de perpetuação da pobreza, com reprodução do cenário de origem ao possibilitar o fechamento de um ciclo de privações materiais, podendo atingir várias gerações.

Pesquisas em âmbito estadual também demonstraram declínio da desnutrição e do retardo estatural em crianças nesta mesma faixa etária em Pernambuco nas últimas décadas ${ }^{(6,7)}$. Comparando-se os resultados encontrados nessa população com a última pesquisa realizada no Estado de Pernambuco ${ }^{(7)}$, observou-se que a prevalência de baixo peso para a idade nas crianças institucionalizadas $(9,6 \%)$ foi três vezes maior que nas crianças de mesma faixa etária na população em geral $(3,2 \%)$. A baixa estatura (12\%), por sua vez, foi 37,9\% maior que o encontrado no inquérito estadual. Com relação aos outros índices antropométricos, observouse neste estudo que a desnutrição, segundo os índices $\mathrm{P} / \mathrm{E}$ $(7,2 \%)$ e IMC/I $(9,0 \%)$, foi cerca de quatro e cinco vezes maior que o relatado na última pesquisa estadual $(1,9 \% \mathrm{e}$ $1,8 \%$, respectivamente) $)^{(7)}$.

Por ter sido utilizada apenas a antropometria, não foi possível a avaliação de carências nutricionais específicas, o que seria interessante em investigações futuras com populações semelhantes. Amostras maiores, análise de informações de várias instituições e coleta de dados adicionais, como a história pré-natal, condições socioeconômicas, de saúde e alimentação das crianças seriam necessárias para uma avaliação da problemática do município. A escassez de estudos semelhantes dificultou a comparação e a discussão dos resultados, e reflete a falta de interesse sobre o tema.

Espera-se que os resultados deste levantamento tragam contribuições para os profissionais que atuam em instituições de acolhimento para crianças e ressaltem a importância de que ações interventivas sejam adotadas para minimizar os efeitos negativos da exclusão social na qual essas crianças estão expostas. Além disso, a realização de estudos prospectivos seria útil para avaliar as consequências futuras de um contexto marcado por uma série de privações sociais e afetivas no período de crescimento e desenvolvimento do indivíduo. Acreditamos que crianças institucionalizadas precisam de atenção especial, sendo necessárias equipes multidisciplinares para o seu atendimento, no intuito de garantir que seu crescimento e desenvolvimento sejam adequados. Portanto, conhecer o perfil dos institucionalizados auxilia no planejamento das unidades de acolhimento, treinamento de funcionários e previsão de gastos para otimização da assistência prestada aos mesmos.

\section{CONCLUSÃO}

A desnutrição e a baixa estatura na amostra estudada apresentaram índices maiores que o encontrado em crianças de mesma faixa etária do Estado de Pernambuco, e estão aquém dos valores aceitáveis por organizações internacionais.

\section{REFERÊNCIAS}

1. Conselho Nacional do Ministério Público (BR). Relatório da Infância e Juventude: resolução $n^{\circ}$ 71/2011: Um olhar mais atento aos serviços de acolhimento de crianças e adolescentes no País. Brasília; 2013 [acesso em 2016 Abr 16]. Disponível em: http://www.cnmp. 
mp.br/portal/images/stories/Destaques/Publicacoes/ Res_71_VOLUME_1_WEB_.PDF

2. Monteiro CA, Benício MHD, Conde WL, Konno SC, Lima ALL, Barros AJD et al. Desigualdades socioeconômicas na baixa estatura infantil: a experiência brasileira, 1974-2007. Estud Av. 2013;27(78):35-49.

3. Correia, LL, Silva AC, Campos JS, Andrade FMO, Machado MMT, Lindsay AC et al. Prevalência e determinantes da desnutrição infantil no semiárido do Brasil. Rev Saúde Pública. 2014;48(1):19-28.

4. Ministério da Saúde (BR). Pesquisa Nacional de Demografia e Saúde da Criança e da Mulher - PNDS 2006: dimensões do processo reprodutivo e da saúde da criança. Brasília: Ministério da Saúde,, 2009. (Série G. Estatística e Informação em Saúde) [acesso em 2016 Abr 16]. Disponível em: http://bvsms.saude.gov.br/ bvs/publicacoes/pnds_crianca_mulher.pdf

5. Oliveira FCC, Cotta RMM, Ribeiro AQ, Sant'Ana LFR, Priore SE, Franceschini SCC. Estado nutricional e fatores determinantes do déficit estatural em crianças cadastradas no Programa Bolsa Família. Epidemiol Serv Saúde. 2011:20(1):7-18.

6. Ministério da Saúde (BR), Instituto Nacional de Alimentação e Nutrição. II Pesquisa Estadual de Saúde e Nutrição: saúde, nutrição, alimentação e condições sócio-econômicas no estado de Pernambuco, Recife. Pernambuco: Secretaria Estadual de Saúde; 1998.

7. Departamento de Nutrição da Universidade Federal de Pernambuco, Instituto Materno Infantil de Pernambuco, Secretaria Estadual de Saúde. III Pesquisa Estadual de Saúde e Nutrição (PESN), Pernambuco, 2006 [acesso em 2016 Abr 16]. Disponível em: http://pesnpe2006. blogspot.com.br/2008/09/sade-e-estado-nutricionalde-crianas-5.html

8. Murta AMG, Lessa AC, Santos AS, Murta NMG, Cambraia RP. Cognição, motricidade, autocuidados, linguagem e socialização no desenvolvimento de crianças em creche. J Hum Growth Dev. 2011;21(2):220-9.

9. Brasil. Lei no .8 .069 , de 13 de julho de 1990 (1990). Estatuto da Criança e do Adolescente [acesso em 2016 Abr 14]. Disponível em: http://www.planalto.gov.br/ ccivil_03/Leis/L8069.htm

10. Cavalcante LIC, Magalhães CMC, Reis, DC. Análise Comparativa do perfil de crianças emacolhimento institucional nos anos de 2004 e 2009. Psico (Porto Alegre). 2014;45(1):90-9.
11. Machado VR. A atual política de acolhimento institucional à luz do estatuto da criança e do adolescente. Serv Soc Rev. 2011;13(2):143-69.

12. Guimarães AF, Carvalho DV, Machado NAA, Baptista RAN, Lemos SMA. Risco de atraso no desenvolvimento de crianças de dois a 24 meses e sua associação com a qualidade do estímulo familiar. Rev Paul Pediatr. 2013;31(4):452-8.

13. Nóbrega JN, Minervino CASM. Análise do nível de desenvolvimento da linguagem em crianças abrigadas Psicol Argum. 2011;29(65):219-26.

14. Baptisa J, Soares I, Henriques M. O impacto da adoção no desenvolvimento da criança. Psicologia. 2013; 27(2):63-79.

15. Ministério da Saúde (BR), Secretaria de Atenção à Saúde, Departamento de Atenção Básica. Orientações para a coleta e análise de dados antropométricos em serviços de saúde: Norma Técnica do Sistema de Vigilância Alimentar e Nutricional - SISVAN. Brasília: Ministério da Saúde; 2011 [acesso em 2016 Abr 10]. Disponível em: http://189.28.128.100/ nutricao/docs/geral/orientacoes_coleta_analise_ dados_antropometricos.pdf

16. Torres CA, Santana JS Neto, Silva MMA, Rossi TRM, Souza LMF, Rocha NSPD. Crianças em passagem: um caminho para a cidadania? Rev Bras Med Fam Comunidade. 2008;3(12):282-9.

17. Chaves, CMP, Lima FET, Mendoça LBA, Custódio IL, Matias EO. Avaliação do crescimento e desenvolvimento de crianças institucionalizadas. Rev Bras Enferm. 2013;66(5): 668-74.

18. Brasil - Ministério da Saúde. Secretaria de Políticas de Saúde. Departamento de Atenção Básica. Saúde da criança: acompanhamento do crescimento e desenvolvimento infantil / Ministério da Saúde. Secretaria de Políticas de Saúde. Brasília: Ministério da Saúde, 2002. Disponível em: http://bvsms.saude. gov.br/bvs/publicacoes/crescimento_desenvolvimento. pdf [Acessado em 15 de abril de 2016]

19. World Health Organization - WHO. Child Growth Standards: Methods and development: Length/heightfor-age, weight-for-age, weight-for-length, weight-forheight and body mass index-for-age. Geneva; 2006.

20. Brasil - Ministério da Saúde. Secretaria de Atenção à Saúde. Departamento de Ações Programáticas Estratégicas. Atenção à saúde do recém-nascido: guia para os profissionais de saúde / Ministério da Saúde, 
Secretaria de Atenção à Saúde, Departamento de Ações Programáticas Estratégicas. - 2. ed. - Brasília: Ministério da Saúde, 2012. volume 1 Disponível em: http://bvsms.saude.gov.br/bvs/publicacoes/atencao_ saude_recem_nascido_profissionais_v1.pdf [Acessado em 15 de abril de 2016]

21. Ferreira FPM. Crianças e adolescentes em abrigos:uma regionalização para Minas Gerais. Serv Soc Soc. 2014;(117):142-68.

22. Monteiro CA, Mondini L, Souza AL, Popkin BM. The nutrition transition in Brazil. Eur J Clin Nutr. 1995;49(2):105-13.

23. Pinho CPS, Silva JEM, Silva ACG, Araújo NNA, Fernandes CE, Pinto FCL. Avaliação antropométrica de crianças em creches do município de Bezerros, PE. Rev Paul Pediatr. 2010;28(3):315-21.

24. Monteiro CA, Conde WL. Secular trends in malnutrition and obesity among children in S. Paulo City, Brazil (1974-1996). Rev Saúde Publica. 2000;34(6):52-61.

25. Silva EC, Eutália ISM, Araújo AC. Síndrome metabólica e baixa estatura em adultos da região metropolitana de São Paulo (SP, Brasil). Ciênc Saúde
Coletiva. 2011;16(2):663-8.

26. Oliveira JS, Lira PIC, Andrade SLLS, Sales AC, Maia SR, Batista-Filho M. Insegurança alimentar e estado nutricional de crianças de São João do Tigre, no semiárido do Nordeste. Rev Bras Epidemiol. 2009;12(3): 413-23.

27. Programa das Nações Unidas para o Desenvolvimento - PNUD, Instituto de Pesquisa Econômica Aplicada IPEA. O índice de desenvolvimento Humano Municipal Brasileiro: atlas do desenvolvimento humano no Brasil [acesso em 2016 Abr 17]. Disponível em: http://www. atlasbrasil.org.br/2013/data/rawData/publicacao atlas_municipal.pdf

27. Araújo ACT, Campos JAD. Subsídios para a avaliação do estado nutricional de crianças e adolescentes por meio de indicadores antropométricos. Aliment Nutr. 2008;19(2): 219-25.

\section{Endereço para correspondência:}

Adriana César da Silveira

Maternidade Escola Assis Chateaubriand - UFC

Rua Coronel Nunes de Melo, s/n

Bairro: Rodolfo Teófilo

CEP 60430-270 - Fortaleza - CE - Brasil 